\title{
THEORETICAL CAPACITY OF MULTI-HOP WIRELESS AD HOC NETWORKS*
}

\author{
Yue Fang \\ RWIN-Lab, Department of Electrical \& Computer Engineering \\ Northeastern University, Boston, Massachusetts, USA \\ yfang@ece.neu.edu
}

\author{
A. Bruce McDonald \\ RWIN-Lab, Department of Electrical \& Computer Engineering \\ Northeastern University, Boston, Massachusetts, USA \\ modonald@ece.neu.edu
}

\begin{abstract}
The capacity of multi-hop wireless ad hoc networks is presented extending the crosslayer model for link capacity in [1]. Two semantics for network capacity are proposed and discussed. The effect of node location on capacity is analyzed within the context of the boundary condition and its impact on evaluation of network capacity. The main focus is on the bottleneck capacity referred to as the "maximum instantaneous network capacity" (MIC). The metric is intended to characterize the true information capacity of the network as a whole-reflecting all possible destinations. The optimization problem is shown to be NP-complete and heuristic algorithms are applied to bound the solution from above. The asymptotic results compare favorably to the well-known results of the highly abstract model in [2].
\end{abstract}

\section{Introduction}

Theoretical models capable of accurate ad hoc network characterization have become increasingly important - robust, efficient and scalable network services depend on understanding the dynamic processes and limitations inherent from these systems. Interest in applications including wireless sensor networks and ubiquitous inter-net access underscores the practical importance of understanding fundamental properties associated with ad hoc network systems (ANS). Capacity bounds and other invariant performance characteristics are crucial elements required for the development of future ANS designed to support real-time and other performance bound applications. A primary obstacle, however, is that ANS exhibit dynamic interactions between entities at different protocol "layers". Hence, these interactions must be understood and integrated into performance analysis and network design.

\footnotetext{
*This work is supported in part by the National Science Foundation under the Faculty Early Career Development <CAREER> Program: NSF Award ID 0347698
} 
The objective of this paper is to present the sequel to the authors' cross-layer analysis of channel capacity in multi-hop ANS. Specifically, a comprehensive investigation focusing on two related approaches to the problem of ad hoc network capacity is presented. The extensibility of the channel model is demonstrated as the previously developed concepts of "the deferral set" and "the equivalent competitor" constitute the basis for an elegant and novel node-oriented model for performance analysis given ideal channel and quasi-static assumptions [1]. The results have interesting theoretical and practical significance with respect to future ad hoc system design — most significant is the parametric model that adapts readily to different MAC protocols and provides direct insight to network design and control problems. This is fundamentally different than previous results that are either too abstract or tightly coupled with specific MAC and routing algorithms. Moreover, under similar constrains the results agree with the well-known bounds in [2], however, provide more practical information and more optimistic results when the constrains are relaxed. Future work will generalize the problem as fundamental limit that is MAC invariant, and present a thorough sensitivity analysis comparing the results to those in [2].

Existing literature focuses primarily on single-hop scenarios or on fully connected networks. Important contributions exist, however, the failure to capture the essence of complex cross-layer interactions and the impact induced by multi-hop environments represent significant shortcomings. With respect specifically to the network capacity problem much of the current literature focuses on simulation results or specific routing protocols. Recently, however, important theoretical results have been reported for multi-hop networks: In [2], the capacity of ad hoc networks is presented with results based on a randomly selected source-destination pair. A significant shortcoming of this model, however, stems from its lack of parametric network characterization. Although the results illustrate multi-hop performance bounds they are derived from highly abstract models. As such, they lack practical insight that can be applied to design more effective networks, which is the objective of cross-layer design. Moreover, the results promote an overly pessimistic vision. Specifically, by failing to account for the benefits of temporal and spatial diversity, coupled with the a non-parametric approach a pre-maturely negative tone emerged in the research community. In contrast, although the assumptions in [4] lack practical relevance, the authors illustrate how diversity, namely, that provided by node mobility can improve the network capacity.

One of the difficulties encountered in the present work is the obtuse nature of the network capacity problem itself. In contrast to channel capacity the definition of network capacity lacks a universal semantic. The ambiguity, however, is used to advantage, namely, by engaging in multiple interpretations more insight is provided. In this paper, network capacity is interpreted in two ways, namely, as (1) "maximum instantaneous capacity" (MIC) and (2) "network saturation capacity" (NSC). The MIC is the maximum amount of data flow in the network at any instant given ideal routing and scheduling; whereas the NSC is the sum of the capacity of all the channels in the network assuming that nodes and traffic are uni- 
formly distributed independent of routing and scheduling algorithms. Both metrics are important for performance analysis, the first is an upper-bound, whereas, the second reflects an achievable flow rate under back-logged conditions.

The remainder of this paper is organized as follows: Section-2 presents analysis of the "network saturation capacity"including characterization and analysis of the "boundary conditions". Section-3 presents alternative approaches for estimating "maximum instantaneous capacity" discussing the merits and shortcomings of each approach. Conclusions are presented in Section-4 elaborating on the contributions and limitations of the results.

\section{Analysis of Network Saturation Capacity}

Without deeper inspection one may mistakenly assume that given an ANS with uniformly distributed nodes, all the "links" will have same capacity, thus, the network saturation capacity is the product of the channel capacity and the number of links in the network. Unfortunately, it is not this simple. Node location, for example, has a direct impact on network capacity. In order estimate network capacity with sufficient precision it is necessary to study the relation between the capacity and node location; one difficulty arises due to the "boundary conditions".

\subsection{Boundary Conditions}

Given a network with $\mathrm{N}$ nodes, each having $n_{\text {avg }}$ neighbors there are $N_{l}=$ $N \times n_{\text {avg }} / 2$ "links". The derivation for arbitrary channel capacity, $S_{\text {chan }}$, is given in [1]. Several examples of NSC (from $n s 2$ simulation using parameters from [1]) are given in Table-1.The table compares simulation results with the $S_{\text {chan }} \times N_{l}$ formulation. In all cases the NSC obtained from simulation are greater than $S_{\text {chan }} \times N_{l}$. The error increases with increasing node density near the boundary (for a fixed network radius). The reason is that the nodes close to the boundary of the network have fewer neighbors, hence, less channel contention. Consequently, in general links close to the boundary have greater available capacity than those in the center of the network. The following definition is required to formalize the problem:

\begin{tabular}{|c|c|c|c|c|c|c|c|}
\hline \multirow{2}{*}{$n_{\text {avg }}$} & \multirow{2}{*}{$N$} & \multicolumn{2}{|c|}{ NSC (Mb/s) } & \multirow{2}{*}{$n_{a v g}$} & \multirow{2}{*}{$N$} & \multicolumn{2}{c|}{ NSC (Mb/s) } \\
\cline { 3 - 4 } & & $N_{l} \times S_{\text {chan }}$ & Simulation & & & $N_{l} \times S_{\text {chan }}$ & Simulation \\
\hline 3 & 49 & 1.75 & 1.779 & 4 & 64 & 1.56 & 1.636 \\
\hline 6 & 81 & 1.06 & 1.52 & 8 & 81 & 0.957 & 1.17 \\
\hline 11 & 75 & 0.417 & 0.86 & 12 & 81 & 0.405 & 0.82 \\
\hline
\end{tabular}

Table 1. NSC: simulation vs. estimation for fixed network radius and transmission range

Definition 2.1 Let $X_{i}$ be a random variable that measures the distance from node $i \in G$ to the network boundary. Assume without loss of generality that transmission is omni-directional and the fixed value $r$ is an accurate estimate of the nominal transmission range given an ergodic, homogeneous network. The boundary zone is defined as the doughnut shaped region occupied by all nodes $i \in G \mid X_{i}<2 r$. 


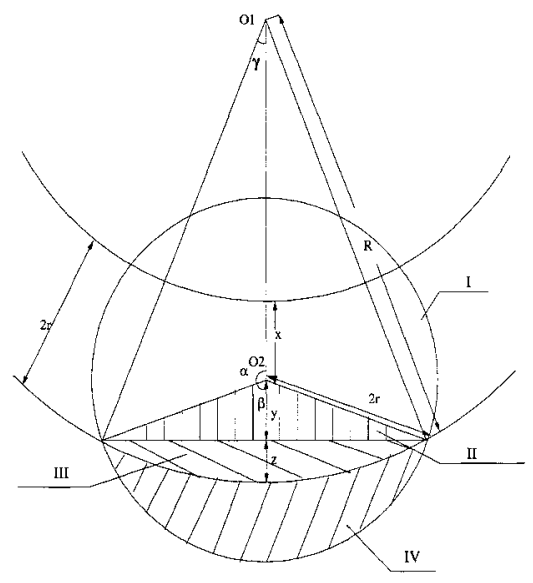

Figure 1. Boundary zone and analysis of boundary condition

The boundary condition quantifies the overestimation of access contention within the boundary zone. Without loss of generality a circular boundary is assumed for the geometric analysis. Figure-1 illustrates the "boundary zone". The arc at the lower part of the figure is the network boundary, while the dashed circle represents the "deferral set" zone ${ }^{1}$ of node $O_{2}$. Observe, however, that for nodes in the boundary zone the physical area covered by the actual deferral set must exclude the shaded area - Area $I V$, which lies outside the network, hence, contains no active nodes. To find the conditions for which the error is negligible assume that the area is occupied by "phantom nodes" that do not produce traffic. The ratio of "phantom nodes" $\left(N_{\text {phantom }}\right)$ to $\mathrm{N}$ should be small. The question is how small for a desired precision? First, it is necessary to find the area of the "phantom zone":

$$
\begin{aligned}
\text { Area }_{I} & =\pi(2 r)^{2} \frac{2 \alpha}{2 \pi} \\
& =4 r^{2} \arccos \left(\frac{8 r^{2}+x^{2}-4 R r-4 r x+2 R x}{4 r(R-2 r+x)}\right) \\
\text { Area }_{I I} & =\frac{1}{2}(2 \cdot 2 r \sin \beta)(2 r \cos \beta) \\
& =2 r^{2}|\sin (2 \alpha)|=4 r^{2} \sin \alpha|\cos \alpha| \\
\text { Area }_{I I I} & =\text { Area of Fan }- \text { Area of Triangle } \\
& =\gamma R^{2}-2 r \sin \beta(R-z) \\
\text { Area }_{I V} & =\pi(2 r)^{2}-\text { Area }_{I}-\text { Area }_{I I}-\text { Area }_{I I I} \\
& =4 \pi r^{2}-4 r^{2} \alpha-2 r^{2}|\sin (2 \alpha)|-\gamma R^{2}-2 r \sin \beta(R-z),
\end{aligned}
$$




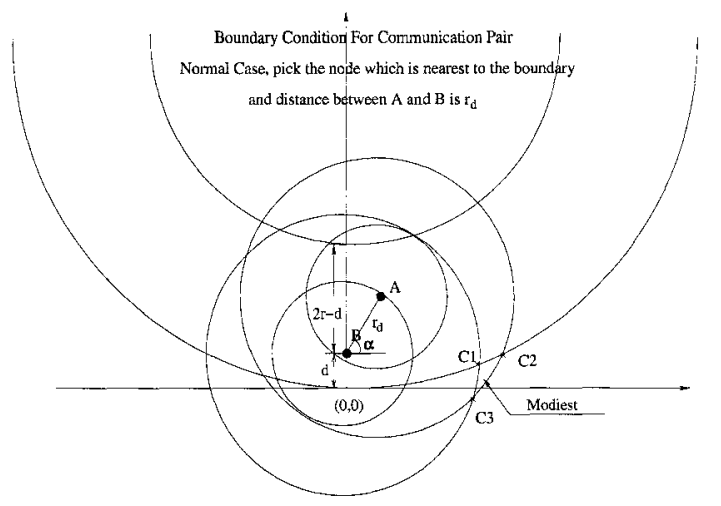

Figure 2. Estimation of phantom zone associated with communicating nodes

where $\alpha, \beta, \gamma, \mathrm{y}$ and $\mathrm{z}$ are shown in Figure- 1 and can be determined geometrically. Figure-2 illustrates the boundary condition with respect to a pair of actively communicating nodes (A,B). The "phantom zone" is the union of Area ${ }_{I V}$ with the parameterized triangular region bounded by $C_{1}, C_{2}$ and $C_{3}$, which is the portion of the zone covered by only one of the nodes (referred to as "Modiest" in the equations). Construct an $\mathrm{x}, \mathrm{y}$ coordinate system with the $\mathrm{x}$ axis tangent to the network boundary and the $\mathrm{y}$ axis perpendicular to the axis at the $\mathrm{x}$ coordinate of the node nearest to the network boundary (node $\mathrm{B}$ in the figure). The coordinates of $C_{1} C_{2}$ $C_{3}$ can be obtained geometrically and the area is calculated as follows:

$$
\begin{aligned}
\operatorname{Area}_{\text {phantom }}\left(d, \alpha, r_{d}\right)= & \operatorname{Area}_{I V}+\operatorname{Modiest}\left(d, \alpha, r_{d}\right) \\
= & \operatorname{Area}_{I V}+\int_{C 3(x)}^{C 1(x)} \int_{d+r_{d} \sin \alpha-\sqrt{4 r^{2}-\left(x-r_{d} \cos \alpha\right)^{2}}}^{d-\sqrt{4 r^{2}-x^{2}}} d y d x+ \\
& \int_{C 1(x)}^{C 2(x)} \int_{d+r_{d} \sin \alpha-\sqrt{4 r^{2}-\left(x-r_{d} \cos \alpha\right)^{2}}}^{R-\sqrt{R^{2}-x^{2}}} d y d x
\end{aligned}
$$

The overestimation of the "equivalent competitors" [1] affecting boundary zone nodes is given by the total number of phantom nodes, $N_{\text {phantom }}$ :

$$
\begin{aligned}
\overline{\mathrm{N}}_{\text {phantom }} & =\rho\left(\int_{0}^{2 r} \frac{1}{2 r}\left(\text { Area }_{\text {phantom }}\left(d, \alpha, r_{d}\right)\right)\right) \\
& =\rho\left(\int_{0}^{2 r} \frac{1}{2 r}\left(\operatorname{Area}_{I V}(d)+\left(\int_{0}^{\pi} \frac{1}{\pi} d \alpha \int_{0}^{r} \frac{1}{r} \operatorname{Modiest}\left(d, \alpha, r_{d}\right) d r_{d}\right)\right) d d\right)
\end{aligned}
$$

The limits of integration reflect the coordinate system and are chosen with respect to the location of the node closest to the network boundary. Thus, the other node must be closer to the center: $\alpha$ varies from 0 to $\pi$ to cover all positions of node $\mathrm{A}$ in this region; the distance $r_{d}$ between nodes $\mathrm{A}$ and $\mathrm{B}$ is varied from 0 to $\mathrm{r}$ reflecting the uniform distribution of nodes. 


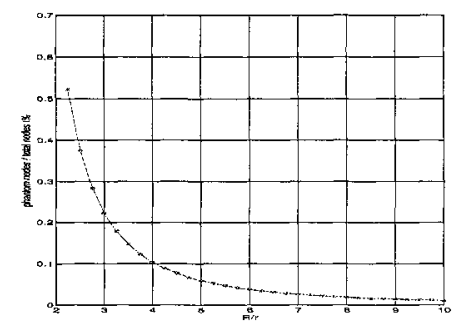

\subsection{Discussion}

Figure 3. $\quad N_{\text {phantom }} / N$ vs $\mathrm{R} / \mathrm{r}$

Numerical analysis depicted in Figure- 3 shows that when the network diameter $(\mathrm{R})$ is on the order of 10 or more times the nominal transmission range $(\mathrm{r})$, the effect of "boundary condition" is less than $1 \%$. This can be regarded as negligible, thus, the NSC can be approximated by the product of $S_{\text {chan }}$ and $N_{l}$. The analysis illustrates how the location of the nodes affects channel capacity, and in turn the NSC. Hence, for moderate to large size networks the boundary effect can be ignored without affecting the accuracy of the NSC.

There are additional parameters that may affect network capacity. The most important of these include: spatial and temporal variation of the distribution of nodes, traffic characteristics, the wireless channel and node mobility. A significant advantage of the present model is that the analysis of these factors is facilitated through probabilistic interpretation of the "equivalent competitor" [1] and enumeration of the effects of the dynamics of the aforementioned parameters. Sensitivity analysis will be included in the extended version of this article.

\section{Analysis of Maximum Instantaneous Capacity}

The analysis of MIC reflects the bottleneck achievable throughput between any set of sources and destinations. Given ideal transmission scheduling it represents a lower-bound on maximum simultaneous flows between all node pairs - given the "ideal scenario" every link must either be transmitting, receiving or in deferral due to the "coupling" effect [3]. Thus, there are a fixed number of links that may be activated simultaneously. The idea is to find a sequence of simultaneously active links that cover the connected network; at each step the number of active links is maximized-the minimum size set represents the desired bottleneck. The shortest covering sequence minimizes the delay as well. The MIC is approximated in two steps. First, find the maximum concurrent active links, second, find the bottleneck of the concurrent active links - the capacity in this case is the MIC.

\subsection{Maximum Number of Simultaneously Active Links}

The first step in solving for MIC is finding the maximum feasible number of concurrent active links. After showing that this problem is NP complete, a suboptimal solution is found using a greedy algorithm. 

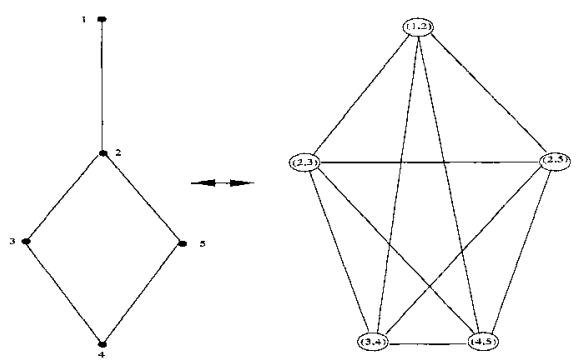

Figure 4. Graph transformation for showing NP-completeness

NP-completeness: Given a network $G(V, E)$ and the definition of deferral link set $D_{\text {Link }}(i, j)^{2}$ [1] associated with link (i,j), consider the following definitions, which are required for the problem formulation:

Definition 3.1 An aggregate deferral link set, $D_{n}$, is defined as a set of deferral link sets associated with a set of simultaneously active links $(i, j)$. The size of $D_{n}$ is equal to the number of deferral link sets in $D_{n}$ and is denoted $S\left(D_{n}\right)$.

$$
D=\bigcup_{(i, j)} D_{\text {Link }}(i, j)
$$

Definition 3.2 Given network $G(V, E)$, an aggregate deferral link set is defined as a deferral partition of $\mathrm{G}: D_{n}=\mathcal{D P}_{n}(G(V, E))$, if and only if it includes all the edges (links) in $G$ :

\section{Problem formulation:}

$$
\bigcup_{(i, j)} D_{\text {Link }}(i, j)=E(G)
$$

Given : $\quad G(V, E)$

Maximize: $S\left(D_{n}\right)$

Over : $\quad$ all $n \mid D_{n}=\mathcal{D} \mathcal{P}_{n}(G(V, E))$

Such that:

$\forall D_{\text {Link }}(i, j) \in D$, and $\left(\forall D_{\text {Link }}(m, n) \in D_{i}\right) \neq D_{\text {Link }}(i, j),(i, j) \ni D_{\text {Link }}(m, n)$

This problem can be reduced in polynomial time to the maximum independent set problem from graph theory ${ }^{3}$, which has been shown to be NP-complete [5] [6]. Thus, the problem of finding the maximum number of simultaneously active links in a network is equivalent to finding the maximum number of independent sets in in the transformed graph $G^{\prime}\left(V^{\prime}, E^{\prime}\right)$. The rules for transforming $G(V, E)$ to $G^{\prime}\left(V^{\prime}, E^{\prime}\right)$ are given as follows (an example is depicted in Figure-4):

- $\forall e \in E$, create a corresponding node n' in graph $\mathrm{G}^{\prime}$ such that $\bigcup n^{\prime}=V^{\prime}$;

- $\forall e \in E$, let $E_{2}(e)=\left\{e_{2} \mid e_{2}\right.$ is less than or equal to two hops away from e $\}$, create a corresponding link e' connects nodes generated by e and every $e_{2} \in E_{2}(e)$ such that $\bigcup e^{\prime}=E^{\prime}$. 


\subsubsection{The Greedy Algorithm}

Heuristic algorithms exist that are efficient and capable of finding optimal solutions to the maximum independent set problem under a well-defined set of conditions. Sufficiently interesting results, however, are attainable for the present capacity problem using a simple iterative greedy algorithm as follows:

\section{Algorithm 3.1}

Step 1: A unique deferral set is associated with each adjacent pair of nodes. A given deferral set is "feasible" if and only if the pair of nodes are not otherwise deferred. List all the feasible deferral sets in ascending order by the number of links in each set.

Step 2: Pick the first deferral set in the list; the corresponding link is assumed to active since its link deferral set has the fewest links. Hence, transmission or flow on the link causes the minimum possible access contention.

Step 3: Update the set of feasible link deferral sets: Sets associated with links within two hops of any active link must be removed from the feasible set because flow on these links will interfere with already active transmissions.

Step 4: Update the size of remaining feasible deferral sets: Care must be taken not to double count any links. Any link that has already been deferred by an active transmission must be removed from any other deferral sets.

Step 5: If more than one deferral set has the same size, the tie is broken by activating the link incident to the pair of nodes with the strongest and most stable signal, or, alternatively, the minimum LOS (line-of-sight) distance.

Step 6: Repeat steps 1-5 until the set of feasible deferral sets is empty.

An example of the results from execution of Algorithm-3.1 is depicted in Figure5. Based on execution of the algorithm there will be 5 simultaneously active links in the network. At any instant when all five links are active all remaining links in the network must defer any attempt to access the transmission medium. Numerical examples of the maximum number of simultaneously active links $\left(l_{\max }\right)$ for different configurations based on algorithm-3.1 are given in Table- 2 .

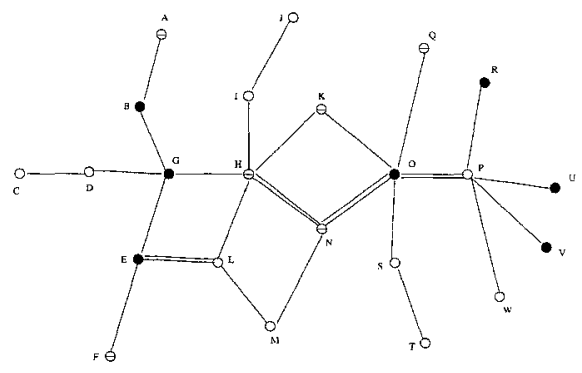

Figure 5. Simultaneously active links using Algorithm-3.1 


\begin{tabular}{|c|c|c|c|c|c|c|c|c|c|c|c|}
\hline $\mathrm{N}$ & $n_{\text {avg }}$ & $l_{\max }$ & $\mathrm{N}$ & $n_{\text {avg }}$ & $l_{\max }$ & $\mathrm{N}$ & $n_{\text {avg }}$ & $l_{\max }$ & $\mathrm{N}$ & $n_{\text {avg }}$ & $l_{\max }$ \\
\hline 432 & 3 & 118 & 530 & 4 & 119 & 732 & 6 & 139 & 952 & 8 & 163 \\
\hline
\end{tabular}

Table 2. Number of simultaneously active links $l_{\max }$ using the greedy algorithm

\subsubsection{The Random Link Selection Algorithm}

A non-determinist algorithm for estimating $l_{\max }$ uses random selection: The algorithm is initialized by placing all the links on the feasible list. At each iteration a link is randomly selected from the feasible list and all the links in its link deferral set are removed from the feasible list. The algorithm iterates until the list is empty.

Random link selection is faster than the greedy heuristic. Moreover, given a uniformly distributed topology of heterogeneous nodes in steady-state the results are of the same order. Given this scenario the number of links is roughly uniform over all the deferral sets other than in the boundary zone. Statistically significant simulation result for random link selection are summarized in Table-3 below:

\begin{tabular}{|c|c|c|c|c|c|c|c|c|c|}
\hline$N$ & $n_{\text {avg }}$ & \multicolumn{4}{|c|}{$l_{\max }$} & \multirow{2}{*}{$N$} & $n_{\text {avg }}$ & \multicolumn{3}{|c|}{$l_{\max }$} \\
\cline { 3 - 8 } \cline { 7 - 10 } & & mean & std & $95 \%$ c.i. & & & mean & std & $95 \%$ c.i. \\
\hline 432 & 3 & 99 & 2.7806 & 1.1125 & 530 & 4 & 87 & 2.4815 & 0.9928 \\
\hline 732 & 6 & 108 & 1.8173 & 0.7271 & 952 & 8 & 115 & 1.7425 & 0.6971 \\
\hline
\end{tabular}

Table 3. Simulation result for random link selection ( 20 independent replications)

\subsection{The Bottleneck Aggregate Link Set}

The first step in finding the maximum instantaneous capacity is to find the maximum possible number of simultaneously active links. This intermediate result is an upper bound that reflects the maximum instantaneous flow. However, in discussing the motivation for this analysis the desired metric was to reflect the bottleneck flow with respect to an arbitrary set of communicating nodes. Hence, MIC must represent the maximum lower bound for the flow of data among arbitrary entities in the network. The optimal solution requires multiple iterations of the independent set problem, hence, it is NP-complete. The following algorithm consists of a polynomial bounded number of iterations of the greedy algorithm:

\section{Algorithm 3.2}

Step 1: Repeat a modified version of Algorithm-3.1 for each link; use the corresponding link to select the initial deferral set and construct a candidate set.

Step 2: Sort the sets by the number of simultaneously active links.

Step 3: Select the largest aggregate deferral set from the candidate list. If all the active links are in the covered link list remove it from the candidate list.

Step 4: Repeat Step-3 until a set is selected with at least one link not in the covered link set.

Step 5: Move the set from the candidate list to the selected list and add any new links to the covered link set.

Step 6: Repeat Steps 2-5 until all the links are in the covered link set. 
At each iteration the algorithm selects the aggregate deferral set from the candidate list that maximizes the number of simultaneously active links so long as it contains at least one active link that has not previously been covered. Thus, the algorithm terminates and provides reachability between all nodes in the connected network. The final iteration represents the bottleneck. Due to the uniformity of the network and the application of the sub-optimal greedy algorithm the upper and lower bounds do not differ significantly. Table-4 shows the simulation result of algorithm-3.1 and 3.2.

\begin{tabular}{|c|c|c|c|c|c|c|c|}
\hline$N$ & $n_{\text {avg }}$ & iterations & lower bound & $N$ & $\overline{n_{\text {avg }}}$ & iterations & lower bound \\
\hline 432 & 3 & 116 & 111 & 530 & 4 & 241 & 110 \\
\hline 732 & 6 & 591 & 128 & 952 & 8 & 1899 & 152 \\
\hline
\end{tabular}

Table 4. Number of required iterations and corresponding lower bound of algorithm-3.1 and 3.2

Algorithm-3.1 and 3.2 provide approximate solutions to the "Maximum instantaneous capacity" problem. However, under ideal conditions it may be possible to achieve better lower bounds, and, thus, show that under worst case analysis it is possible to exceed the results in [2]. Moreover, the solution is not sufficiently efficient. Selected links tend to be "re-activated" in numerous aggregate deferral sets, whereas, other links may be activated only once during the search for the solution. For the purpose of comparison in terms of efficiency and precision a random algorithm is again utilized. Table-5 shows the results. The table shows that the greedy algorithm achieves a tighter lower-bound, however, random selection requires significantly less computation.

\begin{tabular}{|c|c|c|c|c|c|c|c|}
\hline$N$ & $n_{\text {avg }}$ & \multicolumn{3}{|c|}{ iterations needed } & \multicolumn{3}{c|}{ lower bound } \\
\cline { 3 - 8 } & & mean & std & ci (95\%) & mean & std & ci (95\%) \\
\hline 432 & 3 & 45 & 8.474065 & 1.729761 & 94 & 1.295897 & 0.264524 \\
\hline 530 & 4 & 82 & 13.15728 & 2.685719 & 82 & 0.999094 & 0.203939 \\
\hline 732 & 6 & 178 & 21.71956 & 4.433486 & 101 & 1.007220 & 0.205598 \\
\hline 952 & 8 & 341 & 56.42641 & 11.51799 & 107 & 1.559798 & 0.318393 \\
\hline
\end{tabular}

Table 5. Number of required iterations and corresponding lower bound: random algorithm

\subsection{Discussion}

The significance of this work is based on the following observations: (1) In contrast to previous work the semantic of network capacity itself is analyzed in order to provide a clearer understanding and basis for comparison, (2) the results, while sub-optimal, and based on worst-case analysis improve on the most often cited results from [2], (3) the analysis is central of a broad cross-layer framework, hence, it has practical significance with respect to network design, and (4) the underlying models for channel capacity are node-based and parametric, thus they are extensible in terms of access protocols, generalization and application to real control problems as opposed to being based entirely on abstract models. 
In light of the widely accepted limitations and their impact on the broader research community it is critical to examine this work with respect to [2] which studies the capacity of wireless networks. The main results from their analysis relevant to this work is summarized in what follows:

- Throughput is defined as the time average of the number of bits per second that can be transmitted by every node to its destination.

- Capacity is expressed as: $\lambda(N)=\Theta\left(\frac{W}{\sqrt{N \log N}}\right)$ where $\mathrm{W}$ is the channel bandwidth and $\mathrm{N}$ is the number of nodes in the network. Note that capacity has end-to-end significance with respect to a specific source-destination pair.

- Given ideal routing and scheduling their result is shown to improve to $\Theta\left(\frac{W}{\sqrt{N}}\right)$

The network configuration and parameters are the same in both analyses. However, the semantic with respect capacity differs, namely, the MIC analysis is a general bottleneck considering the entire network, whereas, in [2] an upper bound us determined on a per-node basis. This form of result can be misleading as any consumer will experience diminishing returns given a fixed network of resources. The two semantics for capacity in this paper are more useful as they reflect the aggregate scaling effect of the network versus an individual consumer. Furthermore, in [2] the asymptotic results are bounded from above and below. The results in this paper reflect "worst-case" analysis and are bounded only from below. Hence, it can be reasoned that the optimal results are even better.

In order to make a meaningful comparison of the results it is necessary to use information about the present analysis to (1) consider the worst case throughput for a single source destination pair, and, (2) look at asymptotic bounds. From [1] the average area covered by level-1 interference set ${ }^{4}$ is approximately $(\pi+0.98) r^{2}$, thus the number of non-overlapping level-1 interference sets is given by:

$$
\frac{\text { Area }_{\text {network }}}{\text { Area }_{1}}=\frac{\pi}{\pi+0.98} \frac{N}{n_{\text {avg }}+1}
$$

Based on comparison of results from the above estimation and simulation using random link selection it can be shown that the number of non-overlapping level-1 interference sets is roughly equivalent to the number of maximum simultaneously active links. Corresponding asymptotic bounds can expressed as follows:

$$
O\left(\frac{N}{\left(n_{\text {avg }}+1\right)}\right)=\Omega\left(\frac{N}{\left(n_{\text {avg }}+1\right)}\right) \rightarrow \Theta\left(\frac{N}{\left(n_{\text {avg }}+1\right)}\right)
$$

Considering only unicast communications there are at most $\mathrm{N} / 2$ source-destination pairs in the network. The average hop count is approximately $\sqrt{N / n_{\text {avg }}}$. Let $C$ represent a constant that corrects for access capacity $W$ be the channel bandwidth. The resulting capacity per-node is given by (where $C^{t}=2 C$ ):

$$
\lambda(N)=\frac{C \cdot \frac{N}{n_{a v g}+1} \cdot W}{\frac{N}{2} \cdot \sqrt{N / n_{\text {avg }}}}=\frac{C^{\prime} W}{\sqrt{N n_{a v g}}}=O\left(\frac{W}{\sqrt{N}}\right)
$$




\section{Conclusions}

In this paper the authors' previous work [3] [1] has been extended. Building on cross-layer models for link capacity this paper introduces two new metrics for the capacity of wireless ad hoc networks under ideal conditions: "network saturation capacity" and "maximum instantaneous network capacity" are defined and compared. The instantaneous capacity problem reflects the true flow capacity of the network between any nodes-it is the bottleneck capacity, as such it reflects a lower bound on total throughput for all possible destinations. This property differentiates the metric from related work, for example, the asymptotic throughput analyzed in [2]. Determination of the bottleneck capacity is shown to be an NPcomplete problem; two heuristic algorithms are presented for finding approximate solutions. Using the heuristic results and taking limiting values the results that reflect worst-case analysis when bounded from below are shown to agree with the results reported in [2]. The agreement mutually validates the two models, however, it also suggests that the previous work is pessimistic and does not provide insight regarding how to more effectively leverage available network capacity.

\section{Notes}

1. "Deferral set" and "equivalent competitor" in the context characterize the multi-hop wireless network environment and reflect the competition faced by a specific communication, in short, "deferral set" is the union of the nodes which is less than and equal to the two hops away from the active nodes, only transmission originated from those nodes may affect the ongoing transmission, while "equivalent competitors" is the weighted number of nodes in "deferral set", refer to [1] for more details.

2. $D_{l i n k}(i, j)$ is the set of links which are less than or equal to two hops away from link $(\mathrm{i}, \mathrm{j})$, while the size of $D_{\text {link }}(i, j)$ is the number of links in it.

3. An independent set is the largest subset of vertices of $V$ such that no pairs of vertices defines an edge of $E$ for a given graph $\mathrm{G}(\mathrm{V}, \mathrm{E})$.

4. level-1 interference set is the set of direct neighbors of the communication pair

\section{References}

[1] Yue Fang and A. Bruce McDonald, "Theoretical Channel Capacity in Multi-hop Ad Hoc Networks," Proceedings of the 13th IEEE workshop on Local and Metropolitan Area Networks, April 25th-28th, 2004

[2] P. Gupta and P.R. Kumar, "The Capacity of Wireless Networks," IEEE Transactions on Information Theory, vol. 46, no. 2, pp. 388-404, March, 2000

[3] Yue Fang and A.Bruce Mcdonald, "Cross-Layer Performance Effects of Path Coupling in Wireless Ad Hoc Networks: Power and Throughput Implications of IEEE 802.11 MAC," Proceedings of 21 st IEEE International Performance, Computing, and Communications Conference (IPCCC 2002), April 3-5, pp. 281-29, 2002, Phoenix, Arizona

[4] Matthias Grossglauser and David N. C. Tse, "Mobility Increases the Capacity of Ad-hoc Wireless Networks," INFOCOM, pp. 1360-1369, 2001

[5] James Abello, Sergiy Butenko, Panos Paradalos and Mauricio G.C Resende, "Finding Independent sets in a graph using continuous multi-variable polynomial formulations," Journal of Global Optimization, 2001, no. 21, pp. 111-137

[6] Robert Endre Tarjan and Anthony E Trojanowski, "Finding a Maximum Independent Set," SIAM Journal of Computing, September, 1977, vol. 6, no. 3, pp. 537-546, 